\title{
On the Side-Effects of Introducing E-voting
}

\author{
James Heather ${ }^{1}$, Morgan Llewellyn ${ }^{2}$, Vanessa Teague ${ }^{3}$, and Roland Wen ${ }^{4}$ \\ 1 Department of Computing, University of Surrey, Guildford, Surrey GU2 7XH, UK. \\ Email: j.heather@surrey.ac.uk \\ 2 IMT Lucca. Email: hllewell@gmail.com \\ 3 Dept. Computer Science and Software Engineering, The University of Melbourne. \\ Email: vjteague@unimelb.edu.au \\ 4 The University of New South Wales, Sydney. Email: rolandw@cse.unsw.edu.au
}

\begin{abstract}
The literature abounds with discussions on the relative security merits of various voting systems, and on whether a move towards electronic voting is, from a security perspective, something to be encouraged or discouraged. Little has been said, however, on whether there would be unintended side-effects of changing the voting technology, in terms of the votes cast. Security issues aside, should we expect the introduction of an electronic voting system to affect the results of the election?

This paper attempts to tease out some of the possible effects, by analysing ballot data from the 2008 Australian Capital Territory (ACT) Legislative Assembly Election.
\end{abstract}

\section{Introduction}

Significant changes to existing voting technology may produce intended or unintended side-effects on voter behaviour and participation. For instance, in response to the problems with the 2000 Florida presidential election, Florida voting precincts experienced widespread voting technology change from 2000 to 2004 . Results of this change suggest that the change in voting technology was responsible for a $90 \%$ drop in the residual vote rate (that is, the proportion of ballots that were spoilt) during the 2004 election $\left[\mathrm{HPT}^{+} 10\right]$. Similarly, a voting technology change between 2000 and 2004 is attributed to a 35\% drop-off in residual vote rates in Michigan $\left[\mathrm{HPT}^{+} 10\right]$. While debate exists over the significance of the finding, proponents of postal voting often contend this technology increases voter turnout relative to traditional polling station voting.

New voting technologies may also affect voter behaviour by altering perceptions of ballot security and secrecy of the ballot choice. Differences in voter trust, or lack thereof, is often cited as an important consideration when proposing a change in voting technologies [AHT09]. Implementation of a new voting technology without adequate poll worker training may reduce voter evaluations of the voting process [HMP09]. Changes to existing voting technology can also take a partisan tone if ballot order and turnout are affected. It remains an open question if differences in voting technology affect vote choice and turnout decisions. 
Indeed, there are specific instances of election officials implementing a new voting technology in an effort to change voter behaviour. For instance, in the context of Australian elections, a randomized ballot ordering was introduced with the explicit intent of evenly dispersing randomly cast votes across all candidates.

This paper uses data from the 2008 Australian Capital Territory (ACT) Legislative Assembly election to analyze differences in voter behaviour across electronic and paper-based voting technologies. We also draw out several other aspects of voter behaviour from the data.

The results are important in gaining an understanding of how the electoral system can affect the results. This will be of particular interest to researchers who design and build new election technology. For instance, one of the most promising contenders for a secure voting system that could see real world use is Prêt à Voter $\left[\mathrm{RBH}^{+} 09\right]$. Introducing Prêt à Voter into a jurisdiction that currently uses a traditional voting system would require switching to an electronic infrastructure; it would also involve randomizing the order of the names of the candidates on the ballot paper; and it may mean the publication of full (anonymized) ballot data. Would making such changes have unintended sideeffects on voter behaviour?

\section{ACT Legislative Assembly Elections}

The ACT Legislative Assembly is a state-level unicameral parliament with three multi-seat electorates: Brindabella (five seats), Ginninderra (five seats) and Molonglo (seven seats). Members are elected using the Simple Gregory variant of the single transferable vote (STV), which is an electoral system where voters rank the candidates in order of preference, with a minimum of one preference. In a typical election there are 20-30 candidates per electorate, with the major parties being Labor, the Liberals and the Greens.

An unusual feature of ACT Legislative Assembly elections is the use of 'Robson rotation' ballots (see Figure 1). Under Robson rotation, the order of the parties is fixed (chosen by a random draw, independently for each electorate), but the order of candidates within each party is rotated in such a way that there are numerous different versions of each ballot paper (60 for Brindabella and Ginninderra, and 420 for Molonglo). The result of the Robson rotation is that, taken over the entire electorate, no single candidate benefits from random voting (such as simply voting for a party's first $n$ candidates). What is most interesting about Robson rotation is that it was designed to alter voter behaviour; we will discuss this in Section 4.

Also unlike most STV elections in Australia, Legislative Assembly elections do not have group voting tickets, where voters have the additional option of selecting a ticket that corresponds to an ordering of preferences predetermined by groups of political parties and/or independent candidates. Group voting tickets tend to discourage expressivity because voters are more inclined simply to choose the convenience of a group ticket instead of ranking the candidates in the preferred order. For example, in Federal STV elections, over $95 \%$ of voters typically 

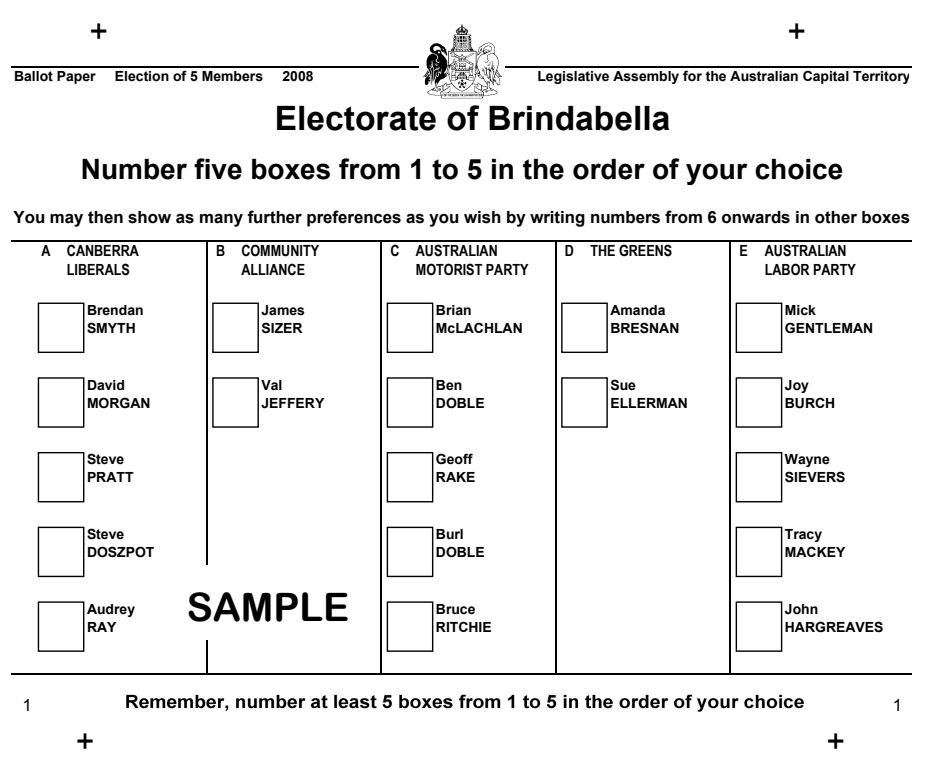

Fig. 1. Sample Ballot Paper for Brindabella

use group tickets. From the point of view of the data analyst, it is helpful that the ACT does not allow group tickets, because they distort the ballot data by encouraging voters to vote for a quick approximation to their true preferences.

Like all elections in Australia, ACT Legislative Assembly elections provide voters with numerous voting options such as pre-poll at major polling places and postal voting. Voters can also vote at any polling place, even outside their home electorate. The ACT is very progressive compared to other Australian jurisdictions in that e-voting has been available as a voting option in some polling stations for the last 10 years.

For the 2001 Legislative Assembly Election, the ACT Electoral Commission developed the Electronic Voting and Counting System (EVACS). The system was initially used on a trial basis but is now widely used on a permanent basis. The e-voting component of EVACS compromises voting machines that display instructions in a choice of languages, and certain machines designed for visually impaired voters have special facilities including large screens, headphones and audio instructions. EVACS voting machines are available in major polling places and can be used by any voter.

EVACS also has an e-counting module that implements the STV counting procedure, which would be extraordinarily difficult to conduct by hand. Paper ballots are scanned with an intelligent character recognition system and manually verified, then uploaded into EVACS and combined with the electronically cast ballots. 
This unique combination of e-voting and e-counting for STV generates extraordinarily useful electronic preferential ballot data. To facilitate statistical analysis, this data contains not only each vote's candidate rankings but also metadata including:

- the Robson rotation ballot positions of the candidates,

- the polling place at which the vote was cast,

- whether the vote was cast electronically or on paper, and

- whether the vote was cast during pre-polling or on election day.

This data is publicly available on the ACT Election Commission website. Our analysis in this paper is drawn from the data for the 2008 Legislative Assembly Election [ACT08].

\section{Party selection and voting interface}

In some polling places, ACT voters were given a choice as to whether to vote electronically or on paper. We analyze voter choices over the voting technology in order to determine if correlation exists between the content of the vote and the method of casting. Of 84 polling places in the ACT 2008 election, five locations (City, Tuggeranong, Belconnen, Gungahlin, Woden) offered the option of electronic voting, to polling day voters and to pre-poll voters. There were no polling places that mandated electronic voting.

Across the 84 polling sites, only $20 \%$ of votes were cast electronically. However, the low rate of electronic voting seems to stem from the fact that only five polling places offered the option of electronic voting rather than voters eschewing electronic devices. In the five locations with electronic voting machines present, approximately $82 \%$ of votes cast were done so electronically $(41,016$ of 50,232 total votes cast). This is a strong indication that, given the choice, ACT voters would choose to use the ACT electronic voting interface rather than paper.

However, the choice of voting interface appears to be correlated with the content of the vote. Table 1 shows the percentage share of the vote for each of the three largest parties (Labor, Liberal, Greens), split by region and by voting method.

The table shows, for each region and each party, the percentage share of the paper votes and the percentage share of the electronic votes; the final column shows the factor by which the vote share changes when moving from paper to electronic (that is, it shows the electronic share divided by the paper share).

It is striking that in every region, the two largest parties, Labor and Liberals, gained a higher share of the paper vote than the electronic vote, whereas the Green vote share is much higher in every region in the electronic ballots than in paper.

\subsection{Analysis}

Perhaps it is not surprising that Green voters are more likely to choose to vote electronically. Familiarity with electronics seems to be negatively correlated with 


\begin{tabular}{|l|l|r|r|r|}
\hline Party & Region & Paper \% & E-vote \% & Factor \\
\hline \multirow{4}{*}{ Labor } & Brindabella & 35.88 & 35.03 & 0.98 \\
\cline { 2 - 5 } & Ginninderra & 41.64 & 38.12 & 0.92 \\
\cline { 2 - 5 } & Molonglo & 35.06 & 34.78 & 0.99 \\
\cline { 2 - 5 } & Overall & $\mathbf{3 7 . 5 8}$ & $\mathbf{3 5 . 7 7}$ & $\mathbf{0 . 9 5}$ \\
\hline \multirow{4}{*}{ Liberal } & Brindabella & 38.86 & 36.55 & 0.94 \\
\cline { 2 - 5 } & Ginninderra & 29.32 & 28.44 & 0.97 \\
\cline { 2 - 5 } & Molonglo & 35.80 & 33.32 & 0.93 \\
\cline { 2 - 5 } & Overall & $\mathbf{3 4 . 2 2}$ & $\mathbf{3 2 . 9 1}$ & $\mathbf{0 . 9 6}$ \\
\hline \multirow{5}{*}{ Green } & Brindabella & 10.24 & 14.63 & 1.43 \\
\cline { 2 - 5 } & Ginninderra & 11.89 & 15.37 & 1.29 \\
\cline { 2 - 5 } & Molonglo & 13.66 & 17.03 & 1.25 \\
\cline { 2 - 5 } & Overall & $\mathbf{1 2 . 2 4}$ & $\mathbf{1 5 . 8 9}$ & $\mathbf{1 . 3 0}$ \\
\hline
\end{tabular}

Table 1. First preferences split by voting interface

age, and Green voters are typically younger. In addition, it is plausible that Green voters may perceive and choose electronic voting as a voting system with a smaller footprint in terms of marginal resource consumption per voter. Furthermore, since the voting machines have special features that encourage usage by voters with visual impairment and voters from a non-English speaking background, there may also be some correlation between these voters and Green voters.

In the ACT, and in Australia as a whole, one might expect the effect of voting technology on the election result to be close to zero. Voting is compulsory in Australia, and one's choice of voting interface should have a negligible affect on the final tally. However, preferential elections suffer from an unusually high rate of invalid voting (roughly $3-4 \%$ in most $\mathrm{ACT}$ elections, which equates to about 3000 votes per seat), primarily due to voter error in marking ballots, for instance through duplicate or missing rankings [HY07]. For voters who choose to vote electronically, such inadvertent errors are eliminated because the EVACS voting machine notifies voters when they attempt to cast an invalid vote. Thus, in the context of electronic voting it is likely that invalid voting is intentional. Since Green voters have a greater tendency to vote electronically, we would expect lower invalid voting rates among Greens, which would effectively increase the overall Green share of the vote.

We predict that this effect would be much more pronounced if a voting system with only an electronic interface were to be introduced in a jurisdiction without compulsory voting. One's decision on whether or not to bother to vote is likely to be influenced by one's level of comfort with the voting interface. We would expect, therefore, an electronic voting system to encourage turnout among Green voters more than among other voters. 
It will be interesting to see whether the same pattern is repeated in the 2012 Legislative Assembly election. If unfamiliarity with electronic systems is a generational phenomenon, the effect will increase as the lower voting age range is filled with technophilic voters and the oldest technophobic voters die; then the effect should stabilize as the first technophiles grow older.

Familiarity with the electronic voting system may also have an effect: if the system was perceived by 2008 voters as easy to use, it might encourage reticent voters to use it in 2012.

\section{Ballot ordering effects}

The organisation of candidate information on ballot papers can influence how voters vote. For example, full-face ballots, which present all the information on a single page, are used almost universally because a ballot paper comprising multiple pages is likely to favour candidates on the first page.

Most jurisdictions worldwide currently require a fixed ballot order for a particular election, consistent across all ballot forms. However, some voting systems, notably Prêt à Voter, mandate a random order of candidates on the ballot paper.

The ACT Legislative Assembly elections are interesting in this regard: the order of the parties is fixed, but the order of candidates within each party is determined by Robson rotation. This makes it possible to analyze the data for ballot ordering effects within parties, by considering whether the votes cast with one order are significantly different from the votes cast with another order; however, because the party order is fixed, it makes it rather harder to glean information as to the effect of the ordering of parties on the ballot paper.

A system like Prêt à Voter is not designed to cope with having several candidates standing for each party, and yet allowing each party's candidates to be grouped together on the ballot paper; in fact, we are not aware of any electronic voting system that allows for this and yet also adheres to the principle of ensuring that the voting terminal does not learn the content of the vote. Enhancing the design of Prêt à Voter to deal with this (not uncommon) situation is worthy of investigation; the results in this section will be informative in assessing the side effects of the various possibilities.

Table 2 shows the vote share achieved by the three main parties when their leaders were, and were not, top of the ballot within their party. The third column shows how many ballots were cast that had a ballot ordering placing the named candidate first within the party, and how many when the named candidate was not listed first; the final column shows the share of the vote received by the party in each case. It will be seen that the effect of the relative ordering of the leader's name has no significant effect on the vote share for that party as a whole. This suggests that ballot order within parties does not significantly affect voters' choice of party. 


\begin{tabular}{|l|c|r|r|}
\hline Candidate & Top? & Ballots & Vote \% \\
\hline \multirow{2}{*}{ Jon Stanhope (Lab) } & Yes & 11980 & 40.7 \\
\cline { 2 - 4 } & No & 48069 & 40.0 \\
\hline \multirow{2}{*}{ Zed Seselja (Lib) } & Yes & 12615 & 31.2 \\
\cline { 2 - 4 } & No & 75651 & 31.6 \\
\hline \multirow{2}{*}{ Meredith Hunter (Green) } & Yes & 29955 & 14.1 \\
\cline { 2 - 4 } & No & 30094 & 13.7 \\
\hline
\end{tabular}

Table 2. Effect on party vote of name ordering

\subsection{Donkey votes}

One natural consequence of compulsory voting is that many people who have no interest in politics or in who wins the election are forced to vote. Some will cast blank or spoilt ballots ('informal votes'); but there is evidence that quite a number of people cast votes that give preferences according to the printed order on the ballot paper, so as to fulfil their legal duties with minimum effort. These votes are termed 'donkey votes'.

A previous study [TEC08] considered some forms of donkey voting in the ACT 1998 election, and concludes that $22 \%$ of votes were donkey votes. However, this study considers only voting behaviour that is indifferent to the ordering of candidates within a party, and does not look at votes that are indifferent to the relative ordering of the parties themselves. A 'linear vote', in the terminology of [TEC08], is one that, for each party, gives its rankings in ascending order down the ballot paper, and so is sensitive to the printed order of the candidates within each party. A 'circular vote' is one that selects a particular candidate within each party, and numbers downwards from that candidate, and then continues the ranking from the top. Both linear votes and circular votes do demonstrate some choice on the part of the voter, and are therefore not full donkey votes.

The ACT uses Robson rotation to counter the effect of linear and circular voting by distributing such votes evenly across all candidates within a party. However, the ordering of parties on the ballot paper is drawn by lots before the election, and, for any given region, is the same for every ballot paper.

It is interesting to ask how many donkey votes there were in the ACT 2008 election. We will consider here only votes that show no clear preference for one party over another.

In addition, in this section we will consider only votes that expressed a full ranking. The reason for this is that these are the clearest candidates for donkey votes. The quickest way to cast a legitimate ballot is to write a ' 1 ' in the top right box and then stop $^{5}$; but since this is a plausible genuine expression of preference, it is impossible to determine which of these votes are donkey votes.

\footnotetext{
${ }^{5}$ Despite the wording on the ballot papers, votes that choose only one candidate are
} treated as formal votes. 
Similar reasoning applies to votes that fill in the first column and then stop. If the party order were randomized on the ballot papers, it would be possible to analyze the effect of the ordering; but unfortunately the ordering is fixed for each region.

Row-monotonic votes A vote is row-monotonic if the projection that maps each ranking to the row in which it appears is monotonic; in other words, the vote is filled in from top to bottom down the ballot paper, but without any constraint on the relative rankings within a row.

There were very few row-monotonic votes in the ACT 2008 election: 4 in Brindabella (of 63,334 ballots in total), 3 in Ginninderra (of 60,049) and 3 in Molonglo (of 88,266). In each region, one of these is also monotonic within each row; that is, the vote is filled in from left to right (or vice versa) across the top row, and then the same across the second row, and so on. (Not all columns are the same length, so not every row has the same number of candidates in it.)

It seems that row-monotonic donkey votes are not a significant problem. This is perhaps because the boxes in each column are nearer together than the boxes within each row, and so it is less effort to fill in the columns one by one. This is where we turn next.

Column-monotonic votes A vote is column-monotonic if the projection that maps each ranking to the column in which it appears is monotonic; in other words, the vote is filled in from left to right or right to left across the ballot paper, but without any constraint on the relative rankings within a column.

In Brindabella, there were 828 column-monotonic votes. Of these, 521 went left to right, and 307 went right to left. These seem natural candidates for donkey votes; the 120 ballots that were fully monotonic (the ranking goes down the first column, then down the second, then down the third, and so on) in particular show strong evidence in this direction.

However, these numbers are in stark contrast to those from Ginninderra and Molonglo. In Ginninderra, there were 16 column-monotonic votes, all of which went left to right; in Molonglo there were 19 that went left to right and 2 that went right to left. Ginninderra and Molonglo each had only 9 fully monotonic ballots.

This gives Brindabella a column-monotonic rate of $1.3 \%$, compared with $0.026 \%$ for Ginninderra and $0.021 \%$ for Molonglo. Why so much higher in Brindabella?

The answer appears to be related to the ordering of the parties on the ballot papers in the three regions. Table 3 below shows the party ordering for each region. The Brindabella party ordering is, purely as a result of the random draw, an ordering that may well fit in with many voters' preferences, either in its listed direction or in reverse. This is all the more so because the Liberal party and the Labor party make a point of instructing their followers (not just in the ACT but nationwide) to put the other last in their ranking, and generally adopt this policy when constructing how-to-vote cards. Such cards are not used in the 


\begin{tabular}{|c|c|c|}
\hline Brindabella & Ginninderra & Molonglo \\
\hline Liberal & Motorists & Pangallo Independents \\
Community Alliance & Labor & Labor \\
Motorists & Community Alliance & Community Alliance \\
Greens & Greens & Richard Mulcahy \\
Labor & Liberal & Motorists \\
& Ungrouped & Liberal Democrats \\
& Ungrouped & Greens \\
& & Liberals \\
& & Ungrouped \\
\hline
\end{tabular}

Table 3. Party order on ballots in each region

$\mathrm{ACT}$; but the thinking may well be adopted by ACT voters in any case. The third largest party, the Green Party, is usually more friendly to the Labor party than to the Liberals, and is in coalition with the Labor party in both the Federal House of Representatives and the Tasmanian Parliament. A Liberal voter who distrusts the Greens and Labor is reasonably likely to vote in the listed order, and a Labor voter who distrusts the Liberals but is amenable to the Greens is reasonably likely to vote in reverse order.

The Ginninderra and Molonglo orderings, however, are much less natural orderings of the parties. In particular, none of the three main parties is listed first or last. A carefully considered column-monotonic vote in Ginninderra or Molonglo would require an idiosyncratic political persuasion, to say the least.

It seems likely, then, that most of the ballots in Brindabella that appear to be full donkey votes are in fact sincerely cast ballots that accord roughly with the voters' preferences. (It is possible that the preference ordering of the two very small parties, the Community Alliance and the Motorists, is still affected by ballot ordering.) This makes for an instructive cautionary tale: if we had only the Brindabella data, we might be all too ready to class all of the fully monotonic ballots as donkey votes.

Moral: think carefully before you call someone a donkey.

\section{$5 \quad$ Expressivity}

ACT Legislative Assembly elections allow voters to express as many preferences as they wish, from choosing a single candidate to giving a full ranking of all candidates. Since STV satisfies the later-no-harm criterion, there is no rational basis for not submitting a full ranking, except if the time cost of filling in the ballot is considered to outweigh the potential benefits of changing the result.

One might therefore hope that a well designed electronic system would encourage voters to be more expressive, and to submit a fuller ranking than they 
would otherwise do. Indeed, a first pass of the 2008 data suggests that there is a small correlation between voting interface and expressivity.

\begin{tabular}{|l|c|l|r|r|}
\hline Region & Seats/Cands & Interface & Votes & Expressivity \\
\hline \multirow{2}{*}{ Brindabella } & \multirow{2}{*}{$5 / 19$} & Paper & 2149 & 7.07 \\
\cline { 3 - 5 } & & Electronic & 11740 & 7.37 \\
\hline \multirow{2}{*}{ Ginninderra } & \multirow{2}{*}{$5 / 27$} & Paper & 3254 & 7.18 \\
\cline { 3 - 5 } & & Electronic & 11244 & 7.59 \\
\hline \multirow{2}{*}{ Molonglo } & \multirow{2}{*}{$7 / 40$} & Paper & 3813 & 9.67 \\
\cline { 3 - 5 } & & Electronic & 18032 & 9.75 \\
\hline
\end{tabular}

Table 4. Number of preferences expressed by paper and electronic voters

Table 4 shows a measure of expressivity for paper and electronic voters in the five regions where both interfaces were offered. The final column shows the mean average number of candidates ranked by the voters. The figures are quite compelling: in each region, the number of preferences given by electronic voters was higher than that given by the paper voters.

There were more candidates in Ginninderra than in Brindabella, which has a small effect on expressivity; but it seems that the number of seats is the key indicator. This is because the majority of voters choose a party, rank the candidates from their chosen party, and then stop; and since the largest parties field the same number of candidates as there are seats, most voters' rankings are the same length as the number of seats.

Alas, this behaviour is what makes the numbers appear more useful than they in fact are. The extra expressivity of electronic voters turns out to be a side effect of the correlation with party choice we have already noted in Section 3. Figure 2 shows, for each possible length of ranking, how many voters cast a ranking of that length. The charts are broken down according to region.

The huge numbers expressing the same number of preferences as there are seats is immediately apparent: the peak dominates the skyline in each case. The second highest peak is consistently for those who diligently give a complete ranking of all candidates. (It is perhaps slightly disappointing from a mathematical perspective that so few voters think to save time and energy by eliding the final preference!)

What next catches the eye are the local maxima evident at 7 (Brindabella and Ginninderra) and 10 (Molonglo). How do we explain these?

Australian politics is dominated by the Labor Party and the Liberal Party. In the previous (2004) election, the Green party had won one seat in Molonglo, and the rest of the seats were shared by Labor (nine) and the Liberals (seven). For this reason, most other parties do not consider it worthwhile to field as many candidates as there are seats. (The only other party to field a full set of 




(a) Brindabella

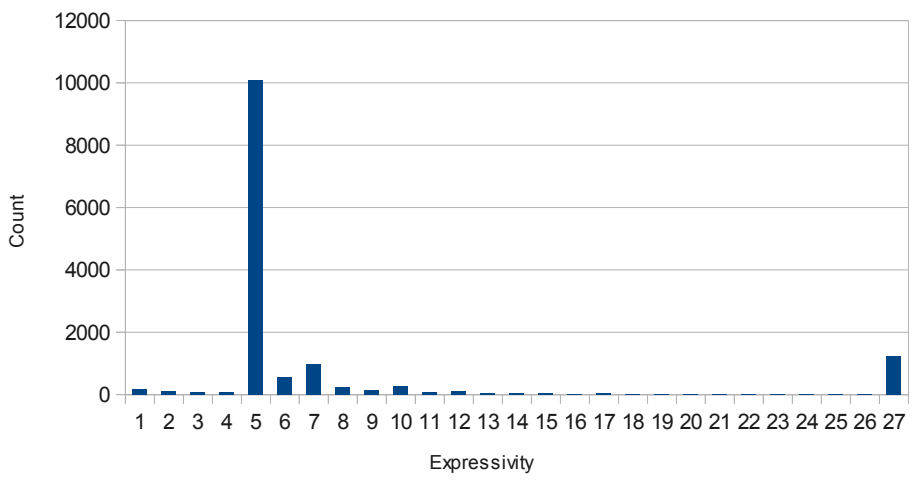

(b) Ginninderra

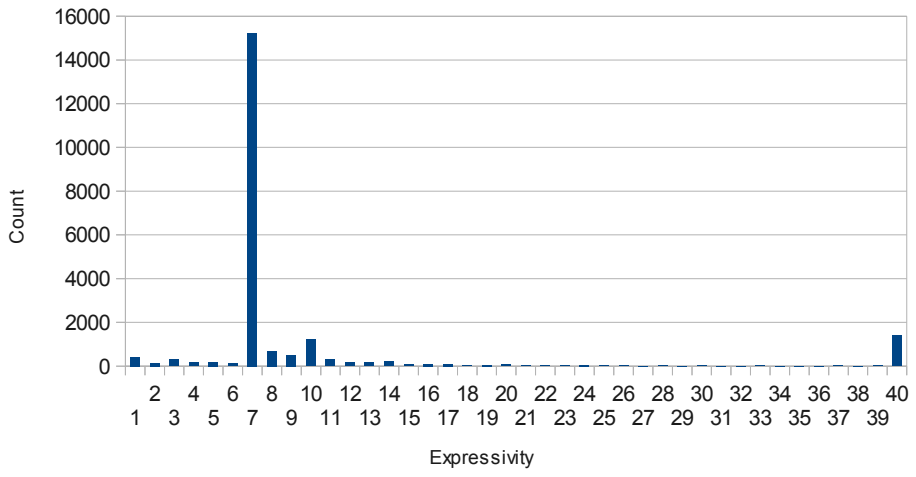

(c) Molonglo

Fig. 2. Expressivity in the ACT 
candidates, even in one region, was the Australian Motorist Party, with around $5 \%$ of first preferences across the ACT.) The Green party thus chose to field two candidates in Brindabella, two in Ginninderra and three in Molonglo.

This explains the local maxima. In each region, the peak comes at the sum $(7 / 7 / 10)$ of the number of seats $(5 / 5 / 7)$ and the number of Green party candidates $(2 / 2 / 3)$. Many of the voters who favour one of the two dominant parties rank the candidates from their party and then stop; a large proportion of Green voters, on the other hand, know that their candidates are less likely to win than those under the Labor or Liberal banner, and so, having ranked the Green candidates in order, they then choose either Labor or Liberal and rank those candidates too.

So Labor and Liberal voters, on the whole, tended to cast rankings of length 5, 5 and 7 in Brindabella, Ginninderra and Molonglo respectively; many Green voters cast rankings of length 7,7 and 10. This gives us the local maxima at $7 / 7 / 10$; but it also explains the greater expressivity in the electronically cast ballots. Voting Green is positively correlated with voting electronically; and voting Green is also positively correlated with greater expressivity.

The ACT data, therefore, does not support the conclusion that electronic voting encourages voters to give a fuller ranking of candidates.

However there are some indications elsewhere in Australia that electronic voting might increase expressiveness. In the 2007 Federal Election, the Australian Electoral Commission trialled two electronic voting systems. One trial used a remote voting system for Australian Defence Force (ADF) personnel deployed overseas and the other used special voting machines for blind and visually impaired (BVI) voters.

Ballot papers in Federal STV elections enable voters to select a group voting ticket rather than marking their own preferences, with the latter commonly referred to as voting 'below-the-line'. Federal elections are very strict in that below-the-line voting requires voters to rank every single candidate (and in many cases there can be around 80 candidates). As a result of these onerous rules, only $3.2 \%$ of voters overall voted below-the-line in 2007 , which is quite typical at Federal level. But post-election evaluations of the trial systems revealed that voters who used the electronic voting systems were more likely to vote this way: $5.2 \%$ in the ADF trial [AEC08b] and 10\% in the BVI trial [AEC08a]. The ADF evaluation found a correlation between network speeds and voting below-theline, and this suggests that voters make a conscious trade-off between the desire to be expressive and the time it takes to cast a vote.

Although no firm conclusions can be drawn from a data sample of this size, it is remarkable that such a high proportion of blind and visually impaired voters who participated in the trial chose to mark all the preferences in spite of the obvious burden in doing so. A possible reason is that BVI voters were making the most of the opportunity to cast a secret ballot: the BVI evaluation found high levels of satisfaction with voter privacy. 


\section{6 'Italian' attacks}

Many secure electronic voting systems require the full ballot data to be published after the election, to enable auditing. Prêt à Voter, for instance, relies for its integrity on publication of both encrypted and unencrypted sets of ballots, with a proof that the sets correspond to the same ballots, but without any information as to the mapping between the sets. Some work has been done on

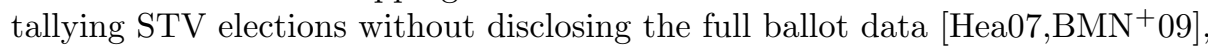
but these solutions are expensive, and if full disclosure can be justified then it is a more efficient option.

One potential weakness of full disclosure is that it allows so-called 'Italian' attacks [Hea07,DC07], where a coercer can bribe and intimidate voters to vote for prescribed candidates. As a simple example, each coerced voter can be instructed to choose a specified candidate as the first preference, then mark the lower preferences so that the ordering forms a uniquely identifiable 'signature'. After the election the coercer uses the electronic ballot data to check which signatures appear, and hence which voters complied with their given instructions. In this way the anonymity of the secret ballot is violated.

How easy is it to mount an Italian attack that will not be obvious from the published ballots? Will such coerced ballots stand out as being atypical voter behaviour?

This is a difficult question to answer definitively, because it depends on what qualifies as 'typical' voter behaviour: what is needed is a coherent and accurate model of how voters fill in their ballots. It may be possible to construct such a model from the ACT ballot data, and this is something that we plan to consider in future work; however, there are some natural options for an Italian attacker that appear attractive with the analysis that we have conducted so far.

The easiest strategy for such an attacker is to specify a sequence of candidates who must be placed at the start of the ranking, and then some random sequence of candidates to follow. This random sequence must be different for each voter whom the attacker wishes to coerce, so that he can determine individual compliance. One might hope that such ballots would be readily identifiable: voters do not cast their ballots randomly, but tend to give similar rankings to similar candidates, and in particular to candidates from the same party.

The ACT ballots do not suggest that these attacks would be easy to spot: there are, in fact, quite a number of voters who vote seemingly randomly. One measure of this is the number of party switches on a ballot - that is, the number of instances of two consecutive preferences that do not select candidates from the same party. And indeed, on this measure, most voters do fill in ballots with a small number of party switches. But a surprising number of voters switch parties at almost every turn.

In Molonglo, for instance, there were a total of 88,266 ballots cast. Most of these were for candidates from a single party, and thus contained no switches. A total of 53,867 ballots contained between one and ten switches. But 63 ballots contained more than thirty party switches; and 7 ballots contained thirty-nine switches. Since there were only forty candidates, this means that seven vot- 
ers took the trouble to construct a ranking that never selected the same party for two consecutive candidates! Similar observations apply to Brindabella and Ginninderra. Some of these are row-monotonic donkey votes discussed in Section 4.1 , but this by no means accounts for all of the ballots with a high number of switches.

There are only two possibilities. Either Italian attacks are already taking place and are present in the ACT data - a fascinating point for further investigation, but it seems unlikely - or such attacks have plenty of room for hiding. An attacker who wants scope for coercing 40,000 voters (quite an operation) could keep all the ballots having at most eight party switches even if he always chose the same eight candidates from which to choose random orderings and they all came from different parties.

It seems likely, then, that without substantially more sophisticated models of voter behaviour, evidence of Italian attacks will be hard to detect from the published ballot data.

\section{Conclusion and future work}

In this paper, we have drawn various lessons from the ACT 2008 ballot data. These lessons are vital considerations when discussing the possibility of changing the electoral system, because it is important to think through not just the primary effects of such changes but also the secondary, often unintentional, effects.

Our ultimate aim is to use ballot data to construct a model of voter behaviour. However, it may well be the case that real world election data is not sufficient to model voter behaviour. Although the ACT data is rich, it does not contain information such as which voters chose a language other than English, or used features for the visually impaired; nor does it log the time at which each ballot was cast. Such fine-grained details would help to rule out certain factors when investigating trends and specific hypotheses, though they are also likely to violate anonymity, and so it seems unlikely that this level of detail will ever be forthcoming for governmental elections. Further studies are needed to determine how to obtain more definitive conclusions.

There appears to be growing interest in electoral systems that allow greater expressivity than that afforded by first-past-the-post: for example, there have recently been (unsuccessful) referenda for the alternative vote in the UK, and STV in Canada. If these systems were to be adopted, then e-voting might have an increased impact on voter behaviour and the election outcome. Possible positive influences might include reductions in invalid voting and donkey voting, while negative influences might include voter confusion due to poor user interface design, which could for instance cause voters to erroneously cast valid votes that are contrary to their intention. The potential for such consequences warrants careful assessment when considering the adoption of e-voting and/or new electoral systems.

More generally, electronic election systems may have already had an impact on increasing expressivity. It would seem that large-scale STV elections are fea- 
sible only with e-counting because the counting procedure is too complex to perform manually. Without e-counting, it is improbable that STV would have been used for Iceland's 2010 Constitutional Assembly, which elected 25 delegates from 525 candidates. In Australia, it appears that STV would not have become so entrenched without e-counting. For example, New South Wales Legislative Council elections use a highly complicated version of STV and have 21 seats, over 300 candidates and 4.5 million voters. Counting the votes manually would simply not be possible.

\section{References}

[ACT08] ACTEC. Ballot paper preference data - 2008 Election, 2008.

[AEC08a] AEC. Final Evaluation Report: Evaluation of the Electronic Voting Trial for Blind and Sight Impaired Electors at the 2007 Federal Election, 2008.

[AEC08b] AEC. Final Evaluation Report: Evaluation of the Remote Electronic Voting Trial for Overseas Based ADF Personnel Electors at the 2007 Federal Election, 2008.

[AHT09] Michael R Alvarez, T E Hall, and A H Trechsel. Internet voting in comparative perspective: the case of estonia. Political Science Politics, 42(03):497$505,2009$.

$\left[\mathrm{BMN}^{+} 09\right]$ Josh Benaloh, T. Moran, L. Naish, K. Ramchen, and Vanessa Teague. Shuffle-Sum: Coercion-Resistant Verifiable Tallying for STV Voting. IEEE Transactions on Information Forensics and Security, 2009.

[DC07] Roberto Di Cosmo. On Privacy and Anonymity in Electronic and Non Electronic Voting: the Ballot-As-Signature Attack. 2007.

[Hea07] James A. Heather. Implementing STV Securely in Prêt à Voter. In Proceedings of the 20th IEEE Computer Security Foundations Symposium, pages 157-169, Venice, Italy, July 2007.

[HMP09] Thad E. Hall, J. Quin Monson, and Kelly D. Patterson. The human dimension of elections: How poll workers shape public confidence in elections. Political Research Quarterly, 62(3):507-522, September 2009.

$\left[\mathrm{HPT}^{+} 10\right]$ Michael J. Hanmer, Won-Ho Park, Michael W. Traugott, Richard G. Niemi, Paul S. Herrnson, Benjamin B. Bederson, and Frederick C. Conrad. Losing fewer votes. Political Research Quarterly, 63(1):129-142, 2010.

[HY07] L. Hill and S. Young. Protest or error? Informal voting and compulsory voting. Australian Journal of Political Science, 42(3):515-521, 2007.

$\left[\mathrm{RBH}^{+} 09\right]$ Peter Y. A. Ryan, David Bismark, James A. Heather, Steve A. Schneider, and Zhe Xia. The Prêt à Voter Verifiable Election System. IEEE Transactions on Information Forensics and Security, 4(4):662-673, December 2009.

[TEC08] TEC. A Discussion Paper on Robson Rotation in Tasmania, 2008. 